\title{
Meta
}

Journal des traducteurs

Translators' Journal

\section{BARAMBONES ZUBIRIA, JOSU (2012): Lenguas minoritarias y traducción: La traducción audiovisual en euskera. Colección TRAMA. Castelló de la Plana: Publicacions de la Universitat Jaume I, 172 p.}

\section{Ana Tamayo}

Volume 59, numéro 1, avril 2014

URI : https://id.erudit.org/iderudit/1026483ar

DOI : https://doi.org/10.7202/1026483ar

Aller au sommaire du numéro

Éditeur(s)

Les Presses de l’Université de Montréal

ISSN

0026-0452 (imprimé)

1492-1421 (numérique)

Découvrir la revue

Citer ce compte rendu

Tamayo, A. (2014). Compte rendu de [BARAMBones ZubiRIA, Josu (2012):

Lenguas minoritarias y traducción: La traducción audiovisual en euskera.

Colección TRAMA. Castelló de la Plana: Publicacions de la Universitat Jaume I,

172 p.] Meta, 59(1), 226-228. https://doi.org/10.7202/1026483ar d'utilisation que vous pouvez consulter en ligne. 
reprises et d'essayer de distinguer ce que nous avons perçu la première fois de ce que nous a apporté le deuxième visionnement.

Bref, si l'étude peut inspirer un chercheur débutant en quête d'un modèle d'étude de cas (celle-ci étant fort bien faite sur le plan méthodologique), sa lecture est fastidieuse pour tout chercheur quelque peu expérimenté, qui n’y trouvera pas son compte, la traduction des films indépendants n'étant visiblement pas un exercice différent au sein de la traduction audiovisuelle.

VALÉRIE FLORENTIN Université Laval, Québec, Canada

Mayra PArra

Université de Montréal, Montréal, Canada

\section{NOTES}

1. Plusieurs études réalisées pour la télévision, dont celle de Media Consulting Group en 2007, Les besoins et les pratiques de l'industrie audiovisuelle européenne en matière de doublage et de sous-titrage, démontrent qu'une émission sous-titrée peut perdre jusqu'à trente pour cent de son audience, les spectateurs n'étant pas habitués au sous-titrage.

2. Entre autres, Halle Berry a eu l'Oscar de la meilleure actrice pour À l'ombre de la haine; Sissy Spacek a eu le Golden Globe de la meilleure actrice pour Sans issue; Nicole Kidman a eu l'Oscar de la meilleure actrice pour Les heures; Éléphant a eu la Palme d'or à Cannes; Traduction infidèle a eu l'Oscar du meilleur scénario original.

3. C'est un fait reconnu et souvent étudié que, le canal visuel demeurant intact (Delabastita 1989), ce sont les autres canaux du texte polysémiotique qu'est le film qui font l'objet d'une adaptation. Les références, notamment culturelles, ne pouvant faire l'objet d'une explicitation vu les contraintes techniques, sont alors adaptées au public (donc, naturalisées). De nombreuses études de cas sont disponibles, dont plusieurs de Teresa Tomaszkiewicz. À l'inverse, une étude plus récente semble vouloir démontrer que les références culturelles sont généralement sauvegardées (Valentini 2011).

4. L'explicitation en traduction est, une fois de plus, une tendance reconnue et largement documentée depuis Blum-Kulka 1986. Par ailleurs, l'explication pragmatique décrite par Pym (1993) n'est rien d'autre qu'une naturalisation.

\section{RÉFÉRENCES}

Blum-KulKa, Shoshana (1986): Shifts of Cohesion and Coherence in Translation. In: Juliane House et Shoshana BLuM-KulKA, dir. Interlingual and Intercultural Communication: Discourse and Cognition in Translation and Second Language Acquisition Studies, 17-35.
Delabastita, Dirk (1989): Translation and Masscommunication: Film and TV Translation as Evidence of Cultural Dynamics. Babel. 35(4): 193-218.

GAmbier, Yves (2002): Les censures dans la traduction audiovisuelle. TTR. 15(2):203-221.

Pettit, Zoe (2004) : The Audio-visual Text: Subtitling and Dubbing Different Genres. Meta. 49(1):25-38.

Pym, Anthony (1993): Epistemological Problems in Translation and its Teaching: A Seminar for Thinking Students. Teruel, Spain: Edicions Caminade.

RAMIÈRE, Nathalie (2004): Comment le sous-titrage et le doublage peuvent modifier la perception d'un film. Meta. 49(1):102-114.

VAlentini, Cristina (2011): La traduction des références culturelles. In: Jean-Marc LAVAur et Adriana Şerban, dir. Traduction et médias audiovisuels. Lille: Septentrion Presses universitaires, 93-110.

BARAmbones Zubiria, Josu (2012): Lenguas minoritarias y traducción: La traducción audiovisual en euskera. Colección TRAMA. Castelló de la Plana: Publicacions de la Universitat Jaume I, 172 p.

Josu Barambones nos introduce en este libro en un campo todavía poco explorado: la traducción audiovisual (TAV) en euskera, lengua sobre la que las investigaciones y publicaciones en este ámbito brillan, aún hoy, por su ausencia. Esta obra resulta tremendamente necesaria, innovadora y útil para los estudios de TAV. Necesaria e innovadora por el ámbito escogido para el estudio: la traducción audiovisual en euskera, una lengua con menos de un millón y medio de hablantes, seis dialectos distintos y aún en proceso de normalización. Se aprecia una notable carencia de estudios descriptivos y empíricos que den cuenta de la situación actual de esta lengua minoritaria y del aporte que la práctica de la TAV ha supuesto en ella, en su cultura y en sus hablantes. Y útil por la metodología utilizada, fácilmente trasladable a otros estudios descriptivos en TAV basados en corpus.

El volumen está estructurado en siete capítulos. Los dos primeros suponen una introducción a la historia y la relación actual que la lengua vasca mantiene con la televisión y la TAV. Los restantes cinco capítulos acercan al lector a la tesis doctoral de Barambones, que, por un lado, pretende arrojar luz sobre las normas y técnicas empleadas en la traducción para doblaje de inglés a euskera y, por otro lado, constituye un análisis contrastivo de los modelos lingüísticos de textos audiovisuales de producción propia en euskera y de producción ajena doblados al euskera. 
En los capítulos introductorios, el autor pone de manifiesto la situación, que dista mucho de ser ideal, de la TAV en euskera y cómo esta realidad se origina a partir de la política lingüística aplicada en el primer canal autonómico vasco (ETB1). Política basada, principalmente, en una lógica lingüística y no televisiva. Barambones no duda en apuntar que la lengua vasca se sostiene, en gran medida, gracias a la traducción. La política lingüística de la cadena vasca ha contribuido a la difusión del euskera unificado, ha cumplido una función educadora y, además, ha logrado una mayor cohesión de la comunidad vascoparlante. Sin embargo, esta política tan conservadora impide que en televisión puedan oírse otros dialectos que se dan de forma natural en el País Vasco y contribuye, como bien apunta el autor, a la poca credibilidad que presentan los productos doblados a esta lengua.

Esta función educadora y didáctica de la cadena en euskera de la televisión pública vasca no queda libre de consecuencias. En primer lugar, priman los productos doblados dirigidos al público infantil, y son pocos los destinados a adolescentes y adultos, por lo que estos últimos no están expuestos habitualmente al doblaje al euskera y, por consiguiente, no otorgan el reconocimiento suficiente a esta modalidad de traducción. En segundo lugar, el euskera es una lengua con pocas variedades lingüísticas en el registro coloquial y, en este sentido, desde el Departamento de Euskera de la ETB se aboga por una traducción en la lengua unificada (o batúa) y en registro estándar que se muestra "desubicada” (Zabalbeascoa 2008) dentro de muchos contextos fílmicos. Por último, escasean tanto la variedad y el registro de voces para doblaje como las producciones originales en euskera, razón por la que no existe un referente lingüístico claro que sirva como modelo de lengua en la práctica de la TAV en euskera. Visto el marco histórico y la situación actual de la TAV en euskera, no cabe duda de que cualquier estudio descriptivo y empírico en el campo resulta innovador y especialmente enriquecedor para aquellos que se dedican tanto a la práctica como a la investigación de la TAV en euskera.

Tras la revisión histórica de los dos capítulos iniciales, Barambones se adentra en la metodología adoptada para el estudio. El capítulo 3 resulta inmensamente útil no solo para aquellos involucrados en la investigación de la TAV en euskera, sino también para cualquiera que investigue en TAV y trabaje con corpus de textos audiovisuales. Los criterios apuntados por el autor para elegir el objeto de estudio responden a razones sólidamente fundamentadas y justificadas, que permiten obtener un corpus realmente relevante para cualquier estudio de TAV. El corpus bilingüe se compone de cinco textos audiovisuales, sus guiones en inglés y sus traducciones para el doblaje en euskera, mientras que el corpus monolingüe lo compone una película de animación de producción original en euskera Karramarro uhartea / La isla del cangrejo (Muñoz y Basterretxea 2000¹). Si bien es cierto que los criterios de selección de ambos corpus están bien fundamentados, se echa de menos un corpus monolingüe algo más extenso, que dé cuenta del modelo de lengua utilizado en otros géneros y que favorezca la solidez de las conclusiones derivadas del análisis contrastivo.

El corpus seleccionado le permite al autor llevar a cabo un análisis descriptivo del corpus textual y un análisis intersistémico que arroja luz sobre los puntos de conexión y divergencia de los modelos de lengua utilizados en las traducciones y en las producciones originales. El análisis descriptivo consiste en (1) la recopilación de datos preliminares sobre cada uno de los textos audiovisuales que conforman el corpus el producto, (2) un estudio macrotextual (con especial hincapié en el análisis de las traducciones de los códigos gráfico y musical) y (3) un análisis microtextual (con especial hincapié en los niveles léxico y morfosintáctico) con base en las técnicas de traducción utilizadas. Barambones engloba los fenómenos encontrados bajo un total de quince técnicas (seis en el nivel léxico y nueve en el nivel sintáctico) basándose en las propuestas de Chaves (2000) y Martí Ferriol (2006). El análisis intersistémico se apoya en la recapitulación de los tres niveles anteriores para llegar a conclusiones sobre el modelo de lengua utilizado en los doblajes al euskera y hacer una comparación entre este y el modelo de lengua observado en el texto original en euskera. Este análisis se lleva a cabo en los niveles fonético, léxico, morfológico y sintáctico.

El último capítulo del volumen presenta las conclusiones extraídas del estudio. La principal conclusión de la primera parte, dedicada a la revisión histórica y a la descripción de la situación actual, es que la política lingüística de ETB1 ha tenido como fin el didacticismo, por lo que escasea la producción de programas destinados a adultos, ejecutado mediante políticas de conservación del idioma y mediante un excesivo control lingüístico, lo que ha generado un estancamiento de la TAV al euskera. El euskera es una lengua aún en proceso de normalización, y la excesiva promoción del batúa, aunque ha supuesto un gran avance en la cohesión cultural y en la normalización y aceptación del idioma, ha frenado el desarrollo de un lenguaje audiovisual funcional, ya que el registro demasiado artificial que se propugna pone en peligro la credibilidad del producto. En cuanto a las conclusiones del análisis descriptivo, Barambones afirma que existen cuatro normas preponderantes en la TAV al euskera, a saber, la 
estandarización lingüística, la naturalización, la explicitación y la elevación lingüística. Por último, la parte más innovadora del presente estudio, la comparación de modelos lingüísticos, corrobora aquello a lo que apuntan la revisión histórica y el estudio descriptivo de las traducciones: el texto original en euskera permite otro tipo de pronunciaciones, más modismos y frases hechas y, en definitiva, está más cerca del lenguaje coloquial porque no está sometido a ningún filtro lingüístico; mientras que el texto traducido se distingue por su formalidad, elevación estilística y cohesión. Cierto es que las traducciones se esfuerzan por acercarse al uso coloquial mediante la utilización, muy puntual, de algunos recursos coloquiales de la lengua vasca, pero estas pinceladas no bastan para dotar al modelo lingüístico de las traducciones de la credibilidad que sí se manifiesta en el lenguaje más dinámico y flexible del texto original.

Para concluir, la obra de Barambones se presenta, no solo como una magnífica obra de consulta para aquellos interesados en realizar estudios de TAV basados en corpus con una potente metodología de corte descriptivista, sino también como un trabajo reivindicativo dentro de los estudios de lenguas minoritarias. Es más, se trata de un volumen que invita a la reflexión sobre la incidencia de aspectos socioculturales en el desarrollo de la traducción para el doblaje y, sobre todo, en el desarrollo de los modelos lingüísticos que imperan en esta modalidad de traducción. La obra, además, constituye el número uno de la nueva colección TRAMA, la primera colección europea que versa exclusivamente sobre el campo de la traducción audiovisual, y a la que le damos la bienvenida desde estas líneas, y deseamos, como investigadores de este campo, que nos siga regalando más volúmenes de la calidad y oportunidad del que aquí reseñamos.

Ana Tamayo

Universitat Jaume I, Castellón, España

\section{NOTAS}

1. Nombre de la película (Año de producción)

\section{REFERENCIAS}

Chaves, Ma José (2000): La traducción cinematográfica. El doblaje. Huelva: Servicio de Publicaciones de la Universidad de Huelva.

Martí Ferriol, José Luis (2006): Estudio empírico $y$ descriptivo del método de traducción para doblaje y subtitulación. Tesis doctoral no publicada. Castellón: Universitat Jaume I. Consultado el 4 de abril de 2014, <http://www. tdx.cat/bitstream/handle/10803/10568/marti. pdf? sequence $=1>$.

Zabalbeascoa, Patrick (2008): La credibilidad de los diálogos traducidos para audiovisuals. In: Jenny Brumme, ed. La oralidad fingida: des- cripción y traducción. Teatro, cómic y medios audiovisuales. Madridù: Iberoamericana/ Vervuert, 157-175.

LARkosh, Christopher (2011): Re-Engendering Translation. Transcultural Practice, Gender/ Sexuality and the Politics of Alterity. Manchester: St. Jerome Publishing, 151 p.

On prête rarement attention aux remerciements de l'auteur ou du directeur d'un ouvrage collectif. Ceux-ci sont pourtant éloquents. Ils donnent le ton à un ouvrage revendicateur mais également intimiste. On retrouve ce même ton dans la première des deux exergues, de Holmes (1970) citant Roland Barthes à propos d'un genre d'écriture qui «...ne traite pas du 'monde' mais des formulations linguistiques faites par d'autres; il s'agit d'un commentaire sur un commentaire». Christopher Larkosh fait toutefois remarquer que son intention derrière ces exergues ne signifie ni un assentiment ni un refus de leur contenu, mais bien l'occasion de susciter un débat.

Précisément d'un débat, celui de la conceptualisation de l'intersubjectivité sexuelle, traitent précisément les textes recueillis par Larkosh et réunis dans l'une des dernières parutions de St. Jerome Publishing.

Dans son introduction, et de retour aux exergues, Larkosh met au jour l'appartenance de Holmes à la communauté gay d'Amsterdam, aspect de la vie du célèbre traducteur et traductologue passé sous silence. Et c'est dans le deuxième exergue qu'il trouve le bien-fondé de sa revendication: «There is so much still to be done!» (Holmes 1984). Par exemple, dépasser les délimitations sexuelles courantes, soit les oppositions binaires conventionnelles, pour aborder des positionnements sexuels jugés ambigus tels que bisexuels, hermaphrodites ou transsexuels. En somme, revisiter la traduction sous l'angle du genre, c'est-à-dire, dans ses termes, "re-engendering translation».

Sans se réclamer de l'exhaustivité ni même de la représentativité, Larkosh réunit ici des travaux qui pointent vers de nouvelles possibilités de recherche, vers une véritable "politique de l'altérité» (p. 7) qui consiste à s'identifier à ceux qui sont différents de nous, voire d'eux-mêmes, et à leur donner publiquement un soutien actif et concret (p. 7).

L'ouvrage comprend sept chapitres de traducteurs et chercheurs issus de diverses traditions culturelles, linguistiques et littéraires. Chaque chapitre, en plus de son titre, s'est vu attribuer un thème sauf le dernier. C'est ainsi qu'on trouve les thèmes suivants: "Writing on race and sexuality in the Harlem Renaissance», "Speaking to the 